\title{
Distribution of interspike times in noise-driven excitable systems
}

\author{
M. C. Eguia and G. B. Mindlin \\ Departamento de Física, FCEN, UBA, Ciudad Universitaria, Pab. I (1428), Buenos Aires, Argentina
}

(Received 2 December 1999)

\begin{abstract}
Noise driven excitable systems present a dynamics which consists of a complex sequence of pulses. In this work we analyze their interspike time distribution, and we find that the organization of the invariant manifolds of the underlying excitable system leaves its fingerprints in it. We derive approximate analytical expressions for the interspike time distribution.
\end{abstract}

PACS number(s): 05.45.-a, 05.40.-a

\section{INTRODUCTION}

A system is called excitable whenever its response to a stimulus is qualitatively different depending on whether the stimulus is smaller or larger than a certain threshold. Excitable systems then present a dynamical stationary state toward which every trajectory eventually tends to after a perturbation: either through a small or large excursion of the available phase space [1]. The paradigmatic example in nature displaying excitable behavior is the neuron. Whenever an excitable system is subjected to noise, the continuous kicking can eventually take the system beyond the threshold, and a spiking will take place. The effects of the random excitation and the deterministic part of the evolution may produce nontrivial behaviors. A natural description of this problem will be in terms of the statistics of times between peaks (likewise, between escapes from the stationary state).

Stated in these terms, noise driven excitable systems can be studied in the framework of what is known as escape problems from metastable states. The seminal contribution in this field has been made by Kramers over 60 years ago [2]. Since then, the emphasis of these studies has been on the computation of the rate of escapes for different potentials (most of them dictated by the particular system under study), subjected to different types of noise (white, colored), also under different approximations [3]. The underlying idea behind those works was that the details of the distributions were consequences of the particularities of the system, and therefore only mean quantities were worth computing. Moreover, only averaged quantities were considered adequate to be compared with experimental data.

Those statements are correct, but recent results have shown that some features of the interspike time distribution contain information on the qualitative properties of the deterministic part of the system: more precisely, the organization of the invariant manifolds of the fixed points of the noise-free case (invariant manifolds are not generally defined for noisy systems) [4]. In a recent work, the bimodal nature of the interspike time distribution for a certain class of noise driven excitable systems was discussed in these terms [5]. In this work we will address this issue. We will study the detailed structure of the interspike time distribution in order to predict the appearance of the two characteristic times and how they depend on the particular features of the system. We also want to unveil whether these characteristic times will be present in other excitable systems. Numerical, analytical tools are required, as well as a good understanding of the underlying deterministic system.

The existence of a noise induced frequency for a noise driven excitable system was first reported, to our knowledge, by Sigeti in 1989 [6]. He studied the Adler equation (an infinite dissipative limit of the equations describing the behavior of pendulum with torque) subjected to noise, and reported the existence of a characteristic time for the escapes from the neighborhood of the stationary state. Several aspects of this phenomenon have been recently described independently by other authors [7]. In the same spirit, Stone and Holmes have described the interspike time distribution of a noise driven system presenting a stable homoclinic connection [8] and derived an analytic expression for the probability distribution. In this work we will study a class of excitable systems having both characteristics mentioned above: excitability and an homoclinic connection for certain parameter values. Hence, we expect to re-encounter the previous distributions as parameters are changed. In a certain region of parameter space the two distributions will coexist, giving rise to the two characteristic times.

This work is organized as follows. Section II is devoted to a fast review of the dynamics of the deterministic pendulum with torque. Although this system is a text example, some of the results will be briefly reviewed to present the notation used throughout the rest of the work. Section III deals with the dynamics found when a pendulum with torque presenting infinite dissipation is subjected to noise. At this point, we will review the classic results by Kramers. The case of finite dissipation will be discussed in Sec. IV. We will study the emergence of another characteristic time in the interspike time distribution. The discussion and the conclusions will be presented in Sec. V.

\section{EXCITABLE SYSTEMS: THE PENDULUM WITH TORQUE}

As mentioned in Sec. I, a system is called excitable if it amplifies inputs whenever these are larger than a given threshold. Then, the ingredients that are necessary to assemble an excitable system are: (a) a stable fixed point, (b) a threshold and (c) a mechanism able to reinject the trajectories crossing the threshold into the neighborhood of the fixed point. A simple way to account for this reinjection is through the topology of the phase space. For example, in $S^{1}$, we simply need a pair of (stable and unstable) fixed points to 
a)

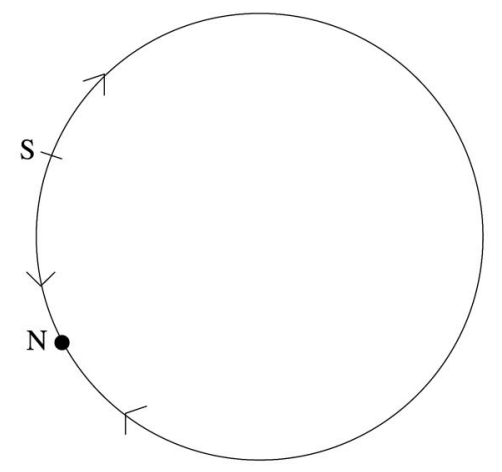

b)

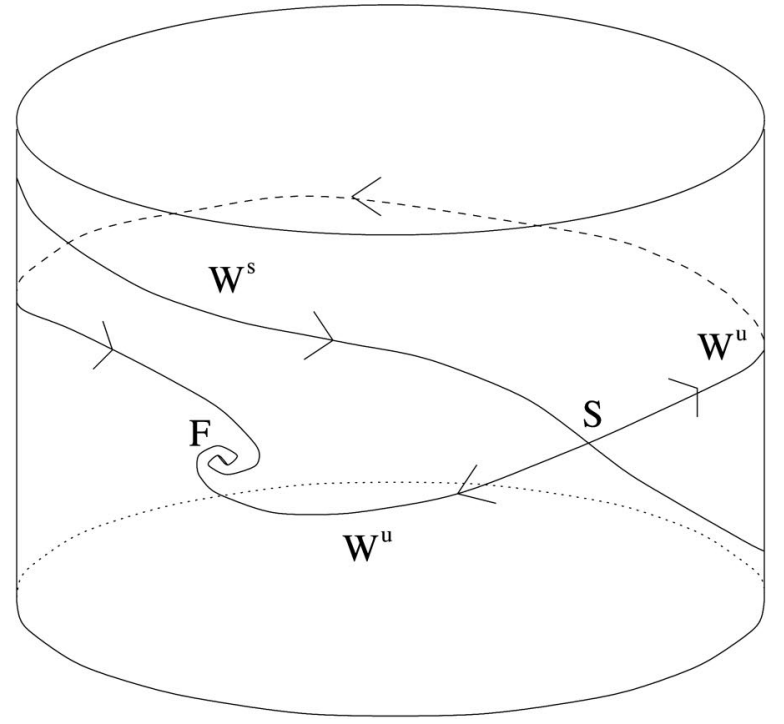

FIG. 1. (a) The simplest excitable system in $S^{1}$ described by Adler equation Eq. (1) with a node $(N)$ and a repulsor $(S)$. (b) Scheme of an excitable system living in $R^{1} \times S^{1}$ (infinite cylinder), with two fixed points: one saddle $(S)$ and one stable focus $(N)$. The unstable manifold of the saddle $\left(W^{u}\right)$ feeds the focus embracing the cylinder.

create an excitable system. This is precisely what we get with the Adler equation

$$
x^{\prime}=\mu-\cos (x) .
$$

When $\mu<1$, we have two fixed points at $x=$ $\pm \arccos (\mu)$. Let us kick a system initially at the stationary solution, with a perturbation larger than the distance between the two fixed points. The trajectory will then evolve returning to the stationary state after completing one full turn to the circle [see Fig. 1(a)]. Sigeti studied the dynamics of this system subjected to noise, and found a maximum in the histogram of the interspike time distribution (in this context, we are calling "spikes" the full turns around $S^{1}$ ). The exponential decay of the distribution, on the other hand, is known since the work of Kramers. The interesting consequence of the existence of a maximum in the interspike time distribution is that even if the system has no periodic orbits, the coupling to a noise source gives rise to a quasioscillatory behavior. This characteristic time can be controlled with the intensity of the noise, and its dispersion has a minimum for a certain noise level. This effect has been recently described as a "coherent resonance" [7].
In a phase space with the topology of the cylinder $R^{1}$ $\times S^{1}$, excitability can also be obtained with two fixed points: a saddle and an attractor. This is displayed in Fig. 1(b). Notice that both branches of the unstable manifold of the saddle feed the attractor, embracing the cylinder. The stable manifold of the attractor (coming now from infinity), creates now the threshold of the excitable system.

In this work, we will show that in bidimensional systems like this, a second characteristic time can arise in the histogram of the interspike time distributions in the form of a second peak. This pseudoperiod is also induced with noise, and is closely linked to the existence of a stable manifold of the saddle point, locally tangent to a linear one associated with a finite (negative) eigenvalue. For this reason this peak cannot be found in the one dimensional system described above, which can be thought of as the infinite dissipation limit of the bi dimensional system.

Both scenarios can be obtained as different limits of the pendulum with torque. Let us assume a simple pendulum of mass $m$, length $l$, in a gravitational field $g$, under the action of a torque $\tau$. Dissipative terms are assumed linear and controlled with the parameter $\gamma$. The dynamical variable $x$ describes the angle with the horizon, and the system is coupled with a thermal bath of temperature $T$. Therefore, the system is ruled by [9]

$$
\begin{gathered}
x^{\prime \prime}+\gamma x^{\prime}+g / l \cos (x)=\tau / m l+\xi(t), \\
\left\langle\xi(t) \xi\left(t^{\prime}\right)\right\rangle=2\left(\gamma K_{B} T / l^{2} m\right) \delta\left(t-t^{\prime}\right),
\end{gathered}
$$

where $\xi(t)$ is a Gaussian noise of zero mean, and $K_{B}$ is Boltzmann constant. Let us call $D=\gamma K_{B} T / l^{2} m, d=g / l$ and $F=\tau / m l$, where we write our systems as

$$
\begin{gathered}
x^{\prime}=y, \\
y^{\prime}=-\gamma y+F-d \cos (x)+\xi(t), \\
\left\langle\xi(t) \xi\left(t^{\prime}\right)\right\rangle=2 D \delta\left(t-t^{\prime}\right) .
\end{gathered}
$$

In Fig. 2 we show the parameter space partitioned in three regions where the dynamics of the noise free system is qualitatively different. In region III we have periodic solutions (the torque is strong enough to force the system to permanently go around $S^{1}$ ). Region II presents an excitable dynamics as the one described at the beginning of the section, with two fixed points (a saddle and an attractor), while region I presents the coexistence of an attractor, a saddle, and an attracting periodic orbit. The separatrix between regions I and II is defined by a homoclinic bifurcation (saddle loop), in which the upper branches of the stable and unstable manifolds coincide. The bifurcation limiting regions II and III is a saddle node, one which has a global connection between the manifolds of the fixed points, in such a way that after the bifurcation a limit cycle remains. Finally, between regions I and III an ordinary saddle-node bifurcation takes place. The three branches meet at the tripartite point saddle-nodehomoclinic bifurcation ( $\mathrm{SNH}$ ) which is a codimension two point. The results that we will present can be generalized to other unfoldings of this codimension two bifurcation. 


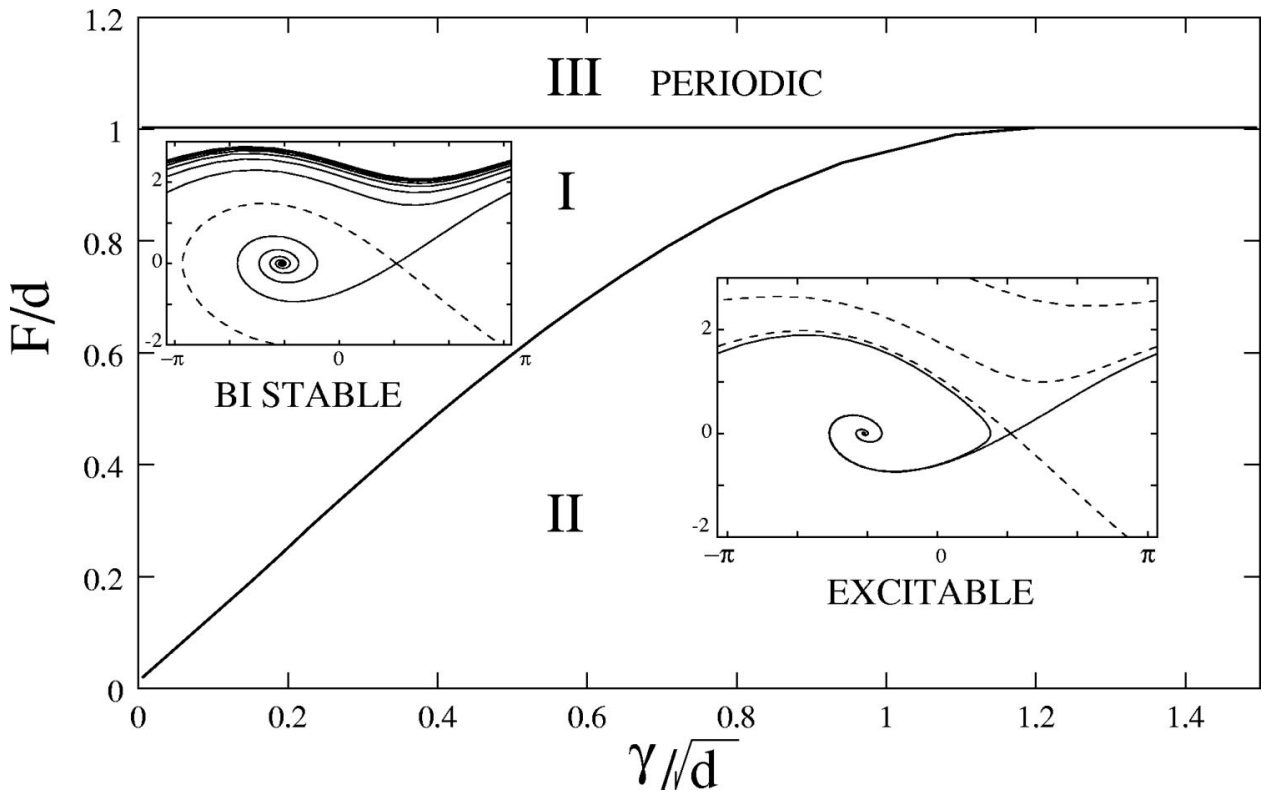

FIG. 2. Bifurcation diagram and phase portraits for the system described by Eqs. (4),(5). In region III we have periodic solutions (the torque is strong enough to force the system to rotate permanently). Region I displays the coexistence of an attractor, a saddle, and an attracting periodic orbit, while region II presents an excitable dynamics. The separatrix between regions I and II is defined by a homoclinic bifurcation, in which the upper branches of the stable and unstable manifolds coincide.

A simulation of the system, Eqs. (4)-(6) is displayed in Fig. 3 for parameter values within region II, near the SNH point. The interspike histogram [Fig. 3(b)] reveals the existence of two different statistical behaviors: an exponential decay predicted by Kramers for long interspike times and a narrow peak for short times. The maximum found by Sigeti is eclipsed by the narrow peak, which is associated to the
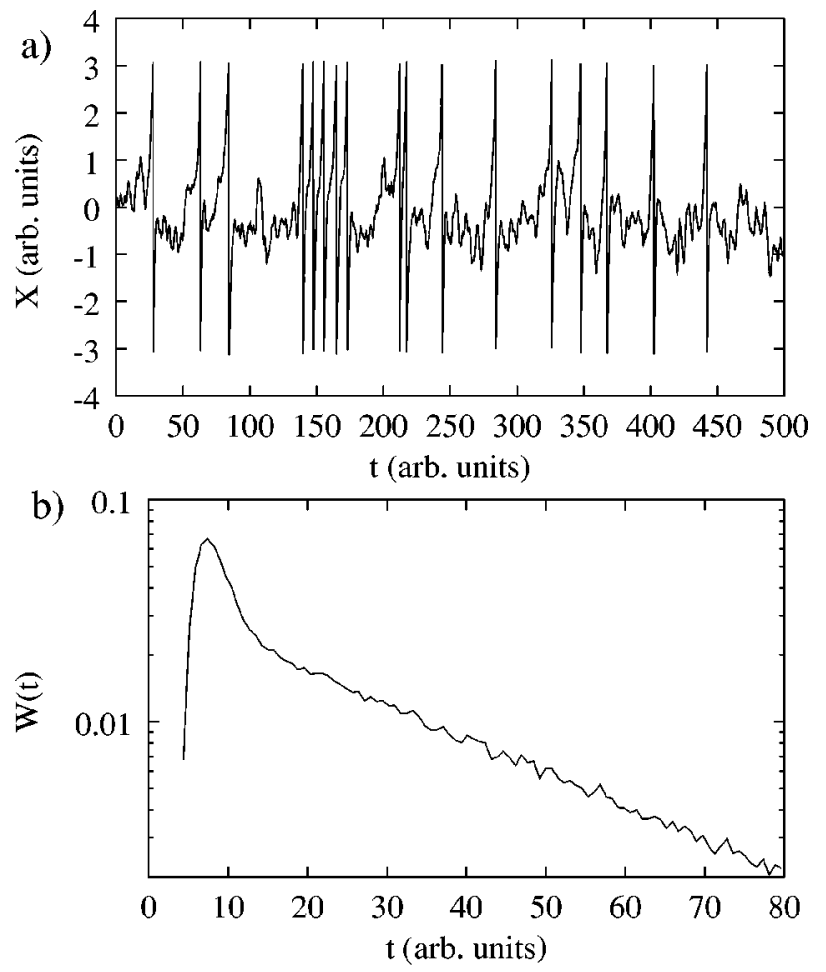

FIG. 3. Temporal series (a) and interspike time distribution (b) for the pendulum with torque [Eqs. (4)-(6)] within the excitable region $(F=0.9, d=1.0, \gamma=1.0$, and $D=0.1)$. The variable $x$ is displayed modulo $2 \pi$, therefore, the jumps of the variable represent full rotations. The left side peak of the histogram corresponds to consecutive spikes in the time series, usually called clusters. There are two characteristic times: the leftmost peak and the exponential decay for larger times. presence of cluster of peaks (C) in the time series [see Fig. $3(\mathrm{a})]$. As the system is moved away from the vicinity of the homoclinic bifurcation we return to the wide-peaked distribution studied by Sigeti. We will start our analysis in following Sec. III with this last distribution, which can be found in the high-friction limit of the bidimensional system. This will allow us to derive an approximate analytic expression for the interspike time distribution.

\section{THE INFINITE DISSIPATION LIMIT}

In the case of large friction, Eqs. (4)-(5) can be written, after the adiabatic elimination of the inertial term, as

$$
x^{\prime}=F / \gamma-(d / \gamma) \cos (x)+\xi(t) / \gamma
$$

Rescaling time $t^{\prime}=t d / \gamma$, and making $\eta\left(t^{\prime}\right)=\xi(t) / d$, this equation reads

$$
x^{\prime}=F / d-\cos (x)+\eta\left(t^{\prime}\right)
$$

Having defined a new time and introducing a new difussion coefficient $R=D / \gamma d$, the correlation function of the noise reads

$$
\left\langle\eta(t) \eta\left(t^{\prime}\right)\right\rangle=2 R \delta\left(t-t^{\prime}\right)
$$

We have then obtained Adler equation, calling $F / d=\mu$. These equations can be thought of as describing the motion of a Brownian particle, in the high friction limit, when subjected to the biased periodic potential: $U(x)=\sin (x)-F x / d$, displayed in Fig. 4.

Now, we are interested in computing, for a set of initial conditions, the distribution of the escape times. Let us begin our program by defining the following expressions, used throughout the rest of this work.

We are calling $P\left(x, t ; x_{0}\right)$ the probability of transition from $x_{0}$ at $t=0$ to $x$ at $t$, which satisfies both the FokkerPlanck (or Kolmogorov) equation 


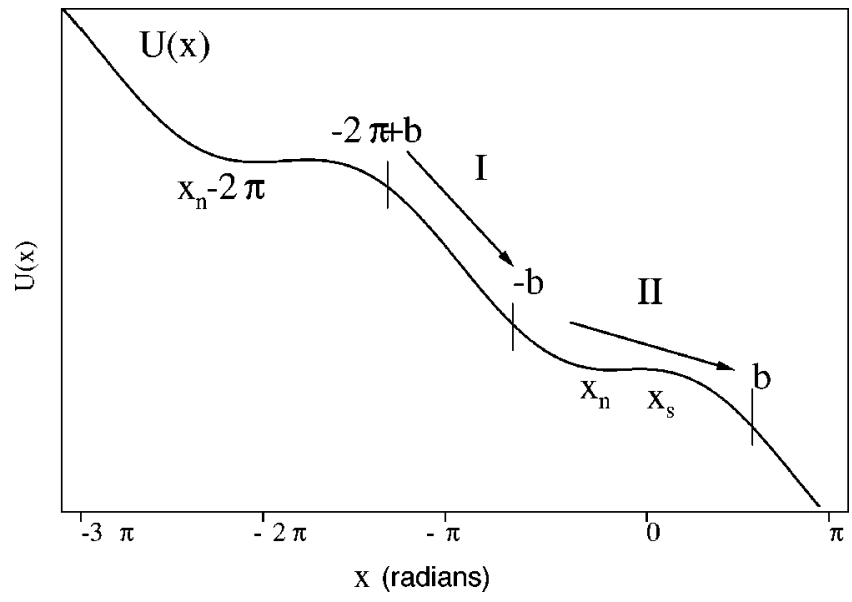

FIG. 4. Potential associated with Eq. (8) interpreted as the highfriction limit of a Brownian particle. The spiking process can be divided in two parts: (I) the reinjection of the trajectories in the box $(-b, b)$ enclosing the saddle $\left(x_{s}\right)$ and the attractor $\left(x_{n}\right)$, and (II) the escape process from this box.

$$
\frac{\partial}{\partial t} P\left(x, t ; x_{0}\right)=-\frac{\partial}{\partial x} F(x) P\left(x, t ; x_{0}\right)+R \frac{\partial^{2}}{\partial^{2} x} P\left(x, t ; x_{0}\right),
$$

with $F(x)=F / d-\cos (x)$, and the backward Fokker-Planck equation (which we will also later use)

$$
\frac{\partial}{\partial t} P\left(x, t ; x_{0}\right)=F\left(x_{0}\right) \frac{\partial}{\partial x_{0}} P\left(x, t ; x_{0}\right)+R \frac{\partial^{2}}{\partial^{2} x_{0}} P\left(x, t ; x_{0}\right) .
$$

Let us denote by $G\left(x_{0}, t ; a, b\right)=\int_{a}^{b} P\left(x, t ; x_{0}\right) d x$ the probability of remaining at the interval $(a, b)$ at time $t$, whenever $x_{0} \in(a, b)$.

Finally, we are calling $W\left(x_{0}, t\right)=-\partial G\left(x_{0}, t ; a, b\right) / \partial t$ the probability density of the escape times from $(a, b)$ for an initial condition in $x_{0} \in(a, b)$.

Even if our system is periodic we will study the distribution of escape times from a box enclosing the saddle $\left(x_{s}\right)$ and the node $\left(x_{n}\right)$, as displayed in Fig. 4. This will allow us to generalize later our results to the case of finite dissipation. Now, we choose the proper boundary conditions. It is easy to see that once that a trajectory goes beyond $x=b$, it is unlikely a return to the box. Therefore, this can be seen as an absorbing wall. On the other hand, trajectories can hardly reach $x=-b$, and if they eventually do, they will return to the neighborhood of the deterministic attractor. Therefore we can call $x=-b$ a reflecting wall. These conditions can be written as

$$
\begin{gathered}
P\left(x=b, t ; x_{0}\right)=0, \\
\frac{\partial}{\partial x_{0}} P\left(x=-b, t ; x_{0}\right)=0 .
\end{gathered}
$$

Unfortunately, the analytical solution for this equation has no closed form. However, it is possible to write an infinite set of recursive equations for the moments of the distribution
$W\left(x_{0}, t\right)$ with closed solutions. The moments can be related to the transition probability through

$$
\begin{aligned}
T_{n}\left(x_{0}\right) & =\int_{0}^{\infty} t^{n} W\left(x_{0}, t\right) d t \\
& =-\int_{0}^{\infty} t^{n} \frac{\partial}{\partial t} G\left(x_{0}, t\right) d t \\
& =-\left(t^{n} G\left(x_{0}, t\right)\right)_{0}^{\infty}+n \int_{0}^{\infty} t^{n-1} G\left(x_{0}, t\right) d t \\
& =n \int_{0}^{\infty} d t \int_{a}^{b} t^{n-1} P\left(x, t ; x_{0}\right) d x,
\end{aligned}
$$

where the integrated term vanishes as long as $G\left(x_{0}, t\right) \rightarrow 0$ (as $t \rightarrow \infty)$, faster than any power. Now, multiplying both sides of Eq. (11) by $n t^{n-1}$, and integrating spatially in the box $(a, b)$ and in time from $t=0$ to $t=\infty$, it is easy to derive (integrating by parts the left side) the following equation for the moments $T_{n}[10]$ :

$$
F\left(x_{0}\right) T_{n}^{\prime}\left(x_{0}\right)+R T_{n}^{\prime \prime}\left(x_{0}\right)=-n T_{n-1}\left(x_{0}\right) .
$$

It is now clear the gain in stating the problem in terms of the moments: the first derivative of the moments will satisfy a first order equation, with closed solutions. Iteratively, we can (in principle) solve as many moments as we need to properly approximate the desired distribution (notice that $T_{0}=1$, since we deal with normalized distributions). All what is left is to translate the original boundary conditions for $P$ into boundary conditions for $T_{n}$. Integrating, we get that $T_{n}(b)=0$ and $T_{n}^{\prime}(-b)=0$. Once these boundary conditions are computed, Eq. (18) can be solved since it is a first order (nonhomogeneous) equation for $T_{n}^{\prime}\left(x_{0}\right)$. Writing $T_{n}\left(x_{0}\right)=u v$ (with $u$ the solution of the homogeneous problem), and solving the two resulting first order equations for $u$ and $v$, one finds that

$$
T_{n}\left(x_{0}\right)=\frac{n}{R} \int_{x_{0}}^{b} d y e^{U(y) / R} \int_{-b}^{y} d z e^{-[U(z) / R]} T_{n-1}(z) .
$$

It is important to keep in mind that the equation under study has been written after rescaling the original time by a factor of $\gamma / d$ for the high-friction limit. Also that, within the same limit, every orbit that leaves our absorbing wall will be reinjected at $x=-b$ (after some mean reinjection time). Therefore, all we are concerned with so far is the set of moments $T_{n}(-b)$.

Even if we have now a prescription for solving our problem, we have to decide whether the integrals written in Eq. (19) will be approximated in order to obtain expressions in closed forms, or if we will choose to lose elegance by computing them numerically. At this point, we find it useful to revisit Kramers' results. The purpose is to illustrate which approximations are incompatible with our objectives.

In the 1940s, Kramers came up with an approximation which allowed him to compute the first moment of the distribution in terms of local properties of the potential in the 
well $\left(x_{n}\right)$ and in the barrier $\left(x_{s}\right)$. The core of this approximation is to convert Eq. (19) to Gaussian integrals. This can be achieved by expanding the argument of the exponential to second order and keeping only the leading terms

$$
\begin{aligned}
T_{1}\left(x_{0}\right)= & \sqrt{\frac{2 \pi}{R U^{\prime \prime}\left(x_{n}\right)} e^{\left(U\left(x_{s}\right)-U\left(x_{n}\right)\right) / R}} \\
& \times \int_{x_{0}}^{b} d y e^{-\left(\left|U^{\prime \prime}\left(x_{s}\right)\right| 2 R\right)\left(y-x_{s}\right)^{2}} \\
= & \frac{\pi}{\sqrt{U^{\prime \prime}\left(x_{n}\right)\left|U^{\prime \prime}\left(x_{s}\right)\right|}} e^{\left(U\left(x_{s}\right)-U\left(x_{n}\right)\right) / R} \\
& \times\left[1-\operatorname{erf}\left(\frac{x_{0}-x_{s}}{l_{h}}\right)\right],
\end{aligned}
$$

where $l_{h}=\sqrt{2 R / U^{\prime \prime}\left(x_{n}\right)}$ is a thermal length. This gives, when $x_{0}=-b$ is far enough from the saddle, and returning to the original variables, the celebrated Kramers' result

$$
T_{\text {Kramers }}=\frac{2 \pi \gamma}{\sqrt{V^{\prime \prime}\left(x_{n}\right)\left|V^{\prime \prime}\left(x_{s}\right)\right|}} e^{(\gamma / D)\left(V\left(x_{s}\right)-V\left(x_{n}\right)\right)},
$$

where $V(x)=U(x) d$ is the real potential.

It is important to notice that, in Kramers' approximation, the mean escape time does not depend on $x_{0}$. It is therefore possible to write, at the same level of approximation, the $n$th moment as

$$
T_{n}=n !\left(\frac{T_{1}}{R}\right)^{n}
$$

Recalling that the moments of the distributions are the Taylor coefficients of the characteristic function (which is related to the probability distribution by means of a Fourier transformation) [11], it is easy to obtain the distribution function predicted within Kramers' framework:

$$
W_{\text {Kramers }}(t)=\frac{1}{T_{1}} e^{\left(-t / T_{1}\right)}
$$

This distribution fits reasonably well the results for long times. Yet, two aspects of the real distribution cannot be captured within this approximation: the impossibility of instantaneous escapes $(W(0)=0)$ and the existence of a maximum. This essential feature of the distribution was missed when we used Gaussian integrals to compute $T_{n}\left(x_{0}\right)$ in Eq. (19).

Going beyond Kramers' result is not trivial. Even when the moments could be calculated using Eq. (19), the reconstruction of the distribution of escape times via the characteristic function converges so slowly that severe numerical problems arise before obtaining a distribution function qualitatively better than Kramers'. Moreover, the series obtained involves cumbersome recurrence formulas and provides no physical insight into the process involved.

Instead, our strategy will consist of first inspecting numerical simulations of our system in order to elucidate the origin of the maximum of the escape-times distribution, and then making a reasonable approximation for the FokkerPlanck equation based on our former observations.

Computing the probability of transition $P\left(x, t ; x_{0}, 0\right)$ within the box $(-b, b)$, it is easy to observe that after a certain time, this distribution reaches a stationary limit (modulo renormalizing the number of particles to account for the escaping ones). The time necessary to reach this "stationary" distribution is approximately the one in which the escape time distribution has a maximum. After this time, the distribution of escape times is approximately exponential (i.e., memoryless, consistent with the observation that the space distribution has reached a stationary state). In other words, the escape process can be understood in two steps: a transient behavior for short times in which the escape times grows rapidly, and a stationary distribution for large times, with a unique characteristic time that can be estimated with Kramers' law.

Kramers' approach seems more natural to explain the exponential tail (actually, Kramers assumed that the metastable state had reached an equilibrium distribution). Now, all that we need is a reasonable approximation for the transient state.

To understand the transient behavior, we will focus on a single realization of the random process. At $t=0$ one particle is injected at $x_{0}=-b$. It will descend, more or less randomly, toward the node at $x=x_{n}$ and eventually climb the potential barrier. If the particle can cross the saddle before the stationary distribution is reached, it will be part of the fast growing part of the distribution of escape times. In the other case, it will be part of the stationary distribution and will obey Kramers' law. We will describe the evolution of the fastest escapes, or equivalently, the transition probability from $x_{0}=-b$ to the top of the barrier for times before the onset of the stationary distribution. The transition probability for fixed spatial coordinates displays a step-like form for obvious reasons: at zero time has zero value and, after a while, it reaches its stationary value. This step function can be seen as a slow "turn on" of the exponential decay. In other words, we are proposing that the distribution of escape times can be understood in terms of a product of two different functions, corresponding to the transient behavior (steplike function) and the stationary one (exponential decay). Now, we attempt to derive the step-like function from the Fokker-Planck equation. Again, if the true transition probability for our potential has no closed form, then we will have to make some approximations.

Let us assume the simplest hypothesis: a linearized flux around the node $x=x_{n}\left(=-x_{s}\right)$. This means that we only deal with first-order dynamics:

$$
\begin{gathered}
x^{\prime}=-\lambda x+\eta(t), \\
\left\langle\eta(t) \eta\left(t^{\prime}\right)\right\rangle=2 R \delta\left(t-t^{\prime}\right),
\end{gathered}
$$

where we shifted the zero of the original $x$ variable to the node $x \rightarrow x+x_{s}$, and $\lambda=U^{\prime \prime}\left(x_{n}\right)$ corresponds to the absolute value of the eigenvalue of the node. The associated FokkerPlanck equation [Eq. (11) with $F\left(x_{0}\right)=\lambda x_{0}$ ] is a typical textbook example [9] (Ornstein-Uhlenbeck process) and has the exact solution 


$$
\begin{gathered}
P\left(x, t ; x_{0}\right)=\frac{1}{\sqrt{2 \pi \sigma(t)^{2}}} \exp \left[-\frac{\left(x-x_{0} e^{-\lambda t}\right)^{2}}{2 \sigma^{2}(t)}\right], \\
\sigma^{2}(t)=\frac{R}{\lambda}\left(1-e^{-2 \lambda t}\right),
\end{gathered}
$$

which, as could be expected, points to a Gaussian distribution with time-dependent mean and standard deviation. In our new variables, the particles are injected at $x_{0}=x_{s}-b$, and we are interested on the transition probability as a function of time, evaluated in a point representing the barrier. In the spirit of Kramers, we chose the point where the potential reaches the height of the barrier $\left(U\left(x_{s}\right)\right)$. Let us call this point $x_{h}$. Then, the probability that a particle injected in $x$ $=x_{0}$ reaches the barrier in a time $t$ is

$$
\begin{gathered}
W_{t r}\left(x_{0}, t\right)=\frac{1}{\sqrt{2 \pi \sigma(t)^{2}}} \exp \left[-\frac{\left(x_{h}-\left(x_{0}+x_{s}\right) e^{-\lambda t}\right)^{2}}{2 \sigma^{2}(t)}\right], \\
x_{h}=2 \sqrt{1-\frac{F x_{s}}{d \lambda}},
\end{gathered}
$$

This is the step function that we have anticipated. We recall now that Kramers' law assumes a stationary distribution and that from our numerical simulations this stationary distribution is only reached after the maximum of the escape time distribution (likewise, the step function). Hence, we can consider the transient distribution [Eq. (27)] and the Kramers' one as two statistically independent processes. Then, we will write our distribution of escape times as a product of the step function and the exponential decay predicted by Kramers

$$
W_{h f}\left(x_{0}, t\right)=W_{0} W_{t r}\left(x_{0}, t\right) e^{-t / \tau_{0}},
$$

where $W_{0}$ is the normalization constant. The characteristic time of the long term decay $\tau_{0}$ still remains an unknown parameter. We have seen that within the Kramers' approach $\tau_{0}=T_{\text {Kramers }}$, but this no longer holds for our distribution function. Thus, we are tempted to return to our moments series [Eq. (19)] in order to derive an expression for $\tau_{0}$. The first moment of the distribution (mean first passage time) will serve our purpose. Again, the computation of the first moment of the distribution of Eq. (29) can be easily done if we approximate the step function [Eq. (27)] by a Heaviside function

$$
\begin{aligned}
T_{1} & =\frac{e^{s / \tau_{0}}}{\tau_{0}} \int_{s}^{\infty} t e^{-t / \tau_{0}} d t \\
& =\tau_{0}+s,
\end{aligned}
$$

where $s$ is the mid point of the step function, and $T_{1}$ is calculated from Eq. (19). We still need an analytic expression for $s$. This can be derived by noting that the step function [Eq. (27)] will reach its mid point when the exponential part of this function does (the prefactor relaxes rapidly to its asymptotic value). We calculate the mid point of the expo- nential in Eq. (27) taking logarithms and calling $z=e^{-\lambda t}$. Then, we obtain the following quadratic equation for $z$ :

$$
\begin{aligned}
{\left[\lambda x_{h}^{2}+\right.} & \left.\lambda\left(b-x_{s}\right)^{2}-2 R \ln (1 / 2)\right] z^{2}+2 \lambda x_{h}\left(b-x_{s}\right) z \\
& +2 R \ln (1 / 2)=0,
\end{aligned}
$$

from which is now easy to calculate $s$ as a function of the parameters of the problem.

Now, we are almost done. In order to finish with the description of the escape process, we have to consider the evolution time interval $\left(t_{0}\right)$ from the saddle point to the absorbing barrier at $x=b$. Since the deterministic escape time from a fixed point goes to infinity we must take into account the noise. We only display the result; the calculation is done in Sec. IV

$$
t_{0} \approx \frac{1}{\lambda} \ln \left(\frac{b-x_{s}}{\sqrt{2 R}}\right)
$$

In Fig. 5 we compare the numerically obtained distributions of escape times from the box $(-b, b)$ for an initial condition in $x=-b$ with the approximate analytical distribution $W_{h f}\left(-b, t-t_{0}\right)$ for three different noise levels. The agreement is quite good. In Fig. 6 we vary the parameter of our system $F / d$ for a fixed noise level. Again, the derived distribution function fits the numerically obtained data. This agreement holds as long as the same hypothesis of the highfriction limit of the Kramers' rate law is maintained. Namely, the barrier height is greater than the noise level and the friction is strong enough. In addition to this, the solution is adequate only when the reinjection point is placed between $-b$ and the node. In any other case the effects of the saddle in the short term are unavoidable. In Sec. IV we will deal with this problem.

\section{THE NOISE DRIVEN PENDULUM WITH TORQUE IN THE CASE OF FINITE DISSIPATION}

In Sec. III we analyzed the interspike time distribution for a pendulum with infinite dissipation. Now we are about to describe how this statistic changes as we begin to lower the dissipation. In order to carry out this program, we are going to review how the organization of the invariant manifolds of the deterministic system changes as this parameter is varied. In Fig. 7, we see three phase space portraits as the dissipation is lowered. The most noticeable feature is that the stable manifolds of the attractor and the saddle approach each other. The distance between the manifolds can become even smaller than the distance between the fixed points.

The dynamical consequence of this process, if the system is coupled to a source of noise, is that the trajectories will find it easier to cross the threshold during the reinjection than during their visits to the neighborhoods of the attractors. These early escapes happen more frequently as the dissipation is lowered. Our previous study was based on the inspection of the trajectories that would begin in the neighborhood of the attractor (or in the phase space region even further away from the threshold than where the attractor was). Now, the issue is precisely how to describe the trajectories that are reinjected eventually close to the threshold. Before attempt- 


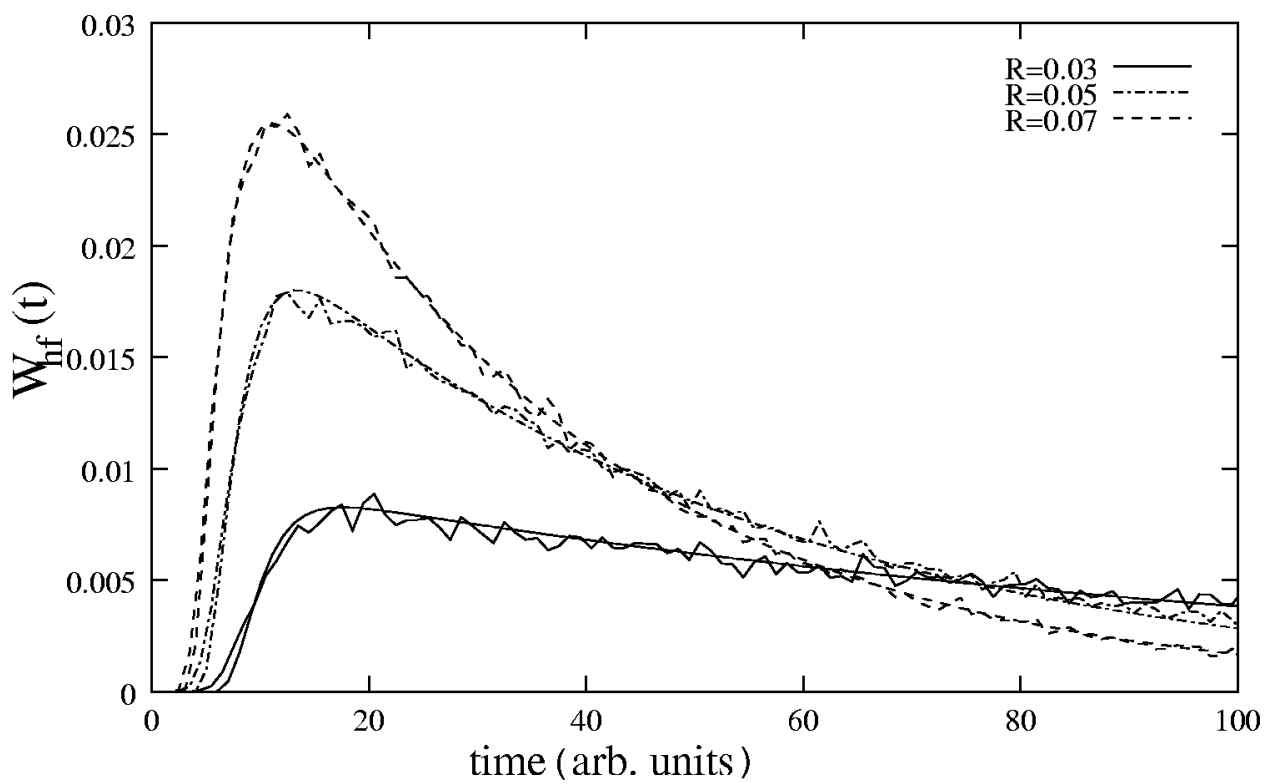

FIG. 5. Distribution of escape times from the attractor in the high friction limit [Eqs. (8),(9)], for initial condition $x(0)=-b$, parameter values $F / d=0.9$, and different noise levels: $R=0.03$ (solid line), $R=0.05$ (dash-dotted line), and $R=0.07$ (dashed line). In each case we compare the result of the numerical simulations (wiggled lines) with the approximated analytical distribution displayed in Eq. (29) (smooth lines). All parameters from Eq. (29) are calculated analytically.

ing a quantitative description of this process, let us note that we expect roughly two families of trajectories: those that after a reinjection come close to the attractor, and those that took advantage of early escapes during the reinjection. Those two will have different statistics. Clearly, the early escapes are associated with small values of interspike times. Notice that although the separation between trajectories in two classes is somewhat arbitrary, the interspike time distributions show two clearly distinct times. As discussed in Sec. I, these look typically like distributions of the kind discussed in Sec. III, superimposed on narrow distributions (corresponding to the early escapes), as can be seen in Fig. 3(b).

In order to unveil the nature of the statistics of the early escapes, we have to study the evolution of trajectories injected close to a saddle, conveniently located at $x=0$. Some of those trajectories are deflected to negative values of $x$, which we can assume will mostly end up visiting the attractor for a while. The others will constitute what we call early escapes, and we are interested in finding the time distribution of those crossing an imaginary box with its rightmost side at $x=\delta$. These will be ruled by the following equations:

$$
\begin{gathered}
x^{\prime}=\lambda x+\eta(t), \\
\left\langle\eta(t) \eta\left(t^{\prime}\right)\right\rangle=2 R \delta\left(t-t^{\prime}\right),
\end{gathered}
$$

which are the same as Eqs. $(23,24)$, but now the OrnsteinUhlenbeck process is unstable. We took advantage of our potential $U(x)$, since the eigenvalues of the fixed points are equal in absolute value. Hence, an initial delta-like distribution at $\left(x_{0}<\delta\right)$ will become a Gaussian distribution with first and second moments increasing exponentially in time. This would be the same as Eqs. $(25,26)$ substituting $t$ by $-t$. Therefore, the probability that at time $t$ the trajectory still remains within a semi-infinite box $(-\infty, \delta)$ is given by

$$
G_{s}\left(x_{0}, t\right)=\frac{1}{\sqrt{2 \pi \sigma^{2}(t)}} \int_{-\infty}^{\delta} \exp \left[-\frac{\left(x-x_{0} e^{\lambda t}\right)^{2}}{2 \sigma^{2}(t)}\right] d x
$$

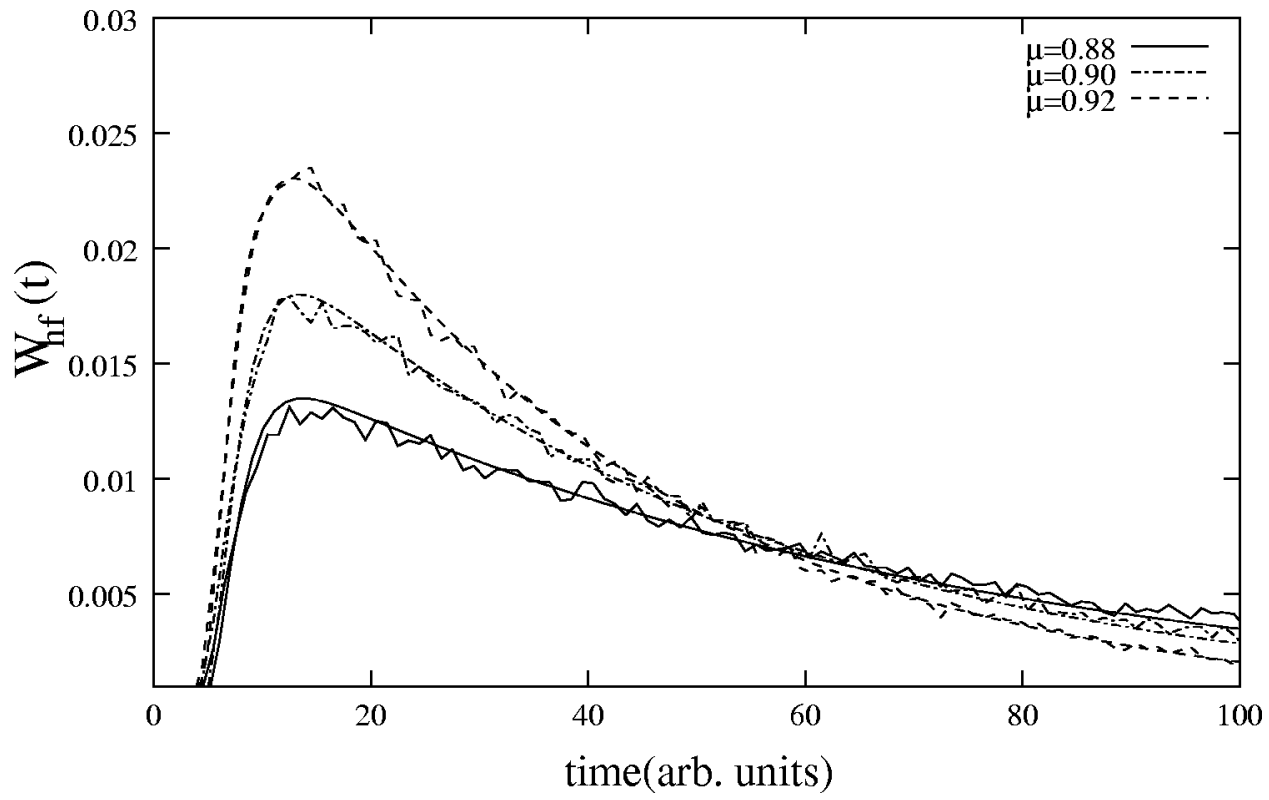

FIG. 6. Distribution of escape times from the attractor in the high friction limit [Eqs. (8),(9)], for initial condition $x(0)=-b$, noise level $R=0.05$ and parameter values: $F / d=0.88$ (solid line), $F / d=0.90$ (dash-dotted line), and $F / d=0.92$ (dashed line). As in Fig. 5 we compare the result of the numerical simulations (wiggled lines) with the approximated analytical distribution displayed in Eq. (29) (smooth lines). 
a)

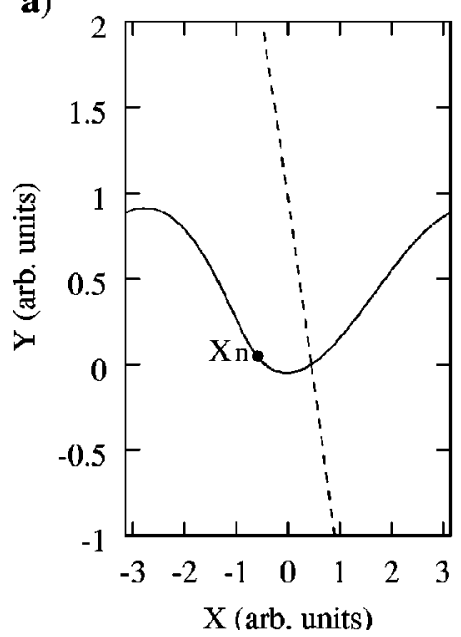

b)

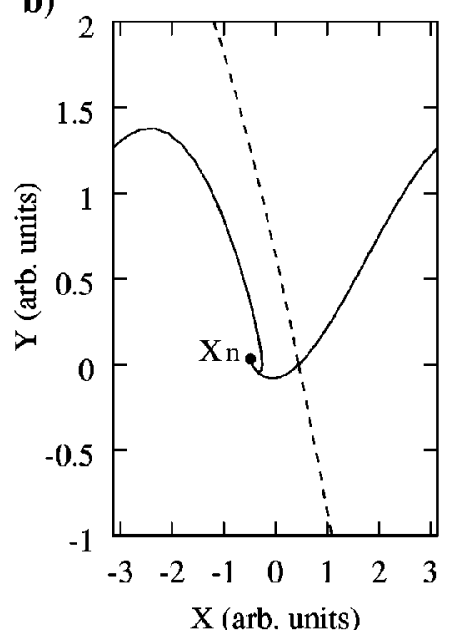

c)

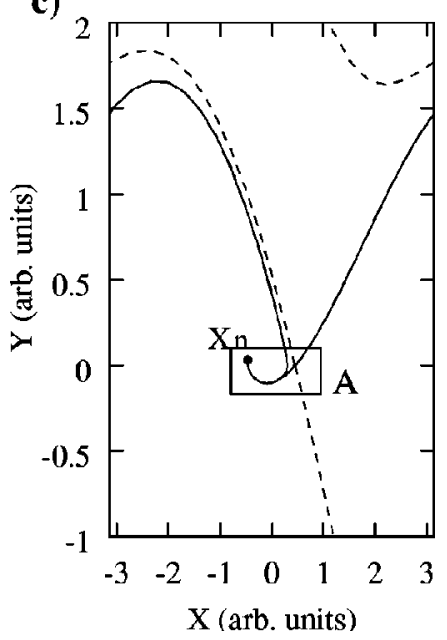

FIG. 7. Phase potratits of the deterministic part of the bidimensional system [Eqs. (4),(5)] for parameter values $F=0.9, d=1.0$, and three friction coefficients: (a) $\gamma=2.0$, (b) $\gamma=1.2$, and (c) $\gamma=0.9$. As the friction is lowered the upper branch of the unstable manifold (solid line) and stable manifold of the saddle (dashed line) approach each other. Box A is used to separate the spiking process (when noise is added) in an escape problem plus a reinjection.

$$
\sigma^{2}(t)=\frac{R}{\lambda}\left(e^{2 \lambda t}-1\right)
$$

which can be properly written, after making $x^{\prime}=(x$ $\left.-x_{0} e^{\lambda t}\right) / \sqrt{2 \sigma^{2}(t)}$ and $\Delta(t)=\left(\delta-x_{0} e^{\lambda t}\right) / \sqrt{2 \sigma^{2}(t)}$ as follows:

$$
\begin{gathered}
G_{s}\left(x_{0}, t\right)=\frac{1}{2}[1+\operatorname{erf}(\Delta(t))] \\
\Delta(t)=\left(\delta-x_{0} e^{\lambda t}\right)\left[\frac{2 R}{\lambda}\left(e^{2 \lambda t}-1\right)\right]^{-1 / 2} .
\end{gathered}
$$

As explained at the beginning of Sec. III, the distribution of escape times is just its time derivative, that is

$$
\begin{aligned}
W_{s}\left(x_{0}, t\right)= & \sqrt{\frac{\lambda^{3}}{2 \pi R}} \frac{\left(\delta-x_{0} e^{-\lambda t}\right)}{\left(e^{2 \lambda t}-1\right)^{3 / 2}} \\
& \times \exp \left[2 \lambda t-\frac{\lambda\left(\delta-x_{0} e^{\lambda t}\right)^{2}}{2 R\left(e^{2 \lambda t}-1\right)}\right],
\end{aligned}
$$

very similar to the expression found by Stone and Holmes when studying the statistics of a noise driven attracting homoclinic loop [8].

We can also make use of this result to calculate the additional time $t_{0}$ added to the distribution in the high-friction limit [Eq. (33)]. The mean first passage time for an initial condition in the saddle going towards the barrier at $x=b$ is

$$
\tau=\int_{0}^{\infty} \operatorname{erf}[\Delta(t)] d t
$$

For low noise level this integral can be approximated to Eq. (33) as shown in [8].

We have then obtained two separate distributions starting from local properties of the system (eigenvalues of the fixed points) and the noise level, which are unable to be deduced only from local properties which weight must be assigned to each distribution. This has to do with the global properties of the system, more precisely with the homoclinic bifurcation described above where the parameter controlling the dissipation is involved. Hence, the relative weight of the distribution will be the only parameter that we will fit. The final distribution of escape times for the one dimensional system, now for an arbitrary reinjection point $x_{0}$, will be a weighted sum of the two distribution deduced above

$$
W_{t o t}\left(x_{0}, t\right)=W_{0}\left[W_{h f}\left(x_{n}, t\right)+c W_{s}\left(x_{0}, t\right)\right],
$$

where $c$ is the relative weight of the distributions. Note that the trajectories that lose this opportunity of an early escape $W_{h f}$ are assumed to start from the node $x_{n}$.

In Fig. 8 we show the distribution of escapes for the one dimensional system [Eq.(8)-(9)] when the trajectories are reinjected in three different points approaching the saddle. We also display our approximated analytic solutions [Eq. (42)] with adjusted weights [Fig. 8(c)] shown in the caption. Again, the agreement is quite good and the functional form obtained easily compares with the distribution of interspike times for the two dimensional system [see Fig. 3(b)].

Now we arrive to the main claim of our work. We argue that the interspike statistics of a two dimensional noise driven excitable system near a saddle-node-homoclinic bifurcation [like the pendulum with torque described by Eqs. (4)(6)] can be reproduced by the escape-time statistics from the metastable state with only one coordinate, endowed with a two dimensional reinjection process. The nontrivial statistics of the interspike histogram generated by the two dimensional system close to the homoclinic bifurcation is mimicked by the one dimensional escape process reinjecting the trajectories close to the barrier. We claim that the escape time distribution of the analogous one dimensional problem can be understood in terms of a superposition of the escape-time distribution in the high-friction limit $W_{h f}(t)[\mathrm{Eq} .(8)]$ and the distribution of early escapes $W_{s}(t)$ [Eq. (40)].

The distribution of escape times $W_{t o t}\left(x_{0}, t\right)$ will reproduce the interspike histogram for the two dimensional sys- 


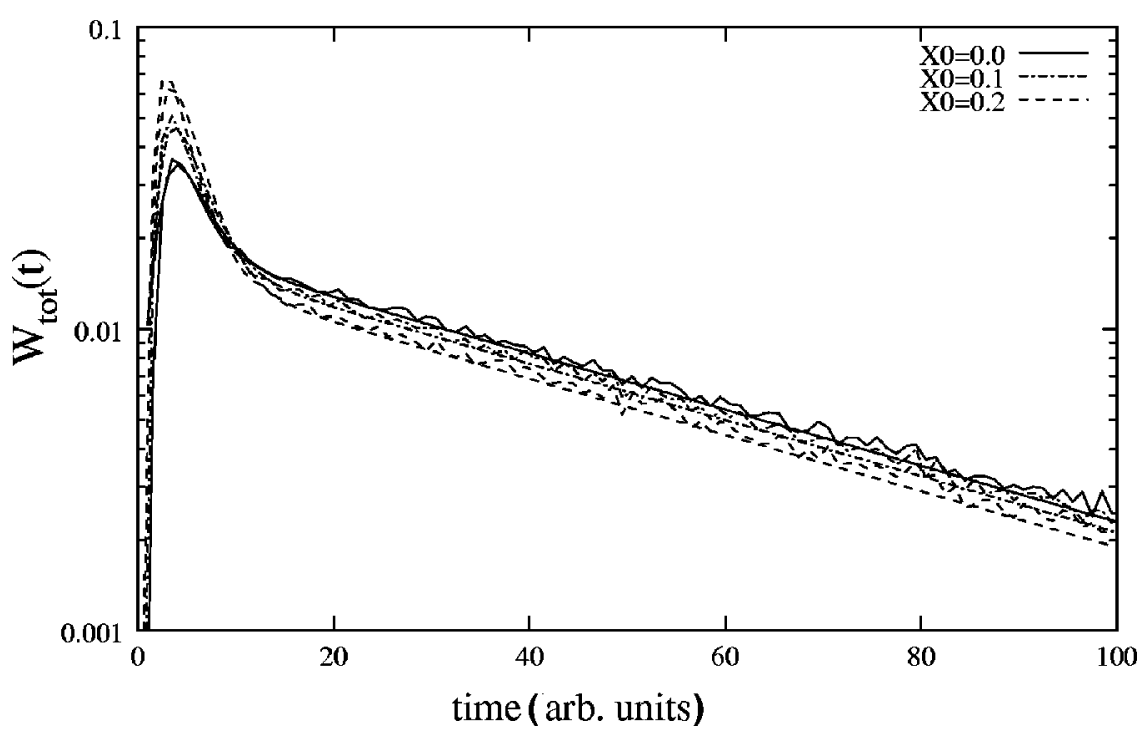

FIG. 8. Distribution of escape times from the attractor in the high friction limit [Eqs. (8), (9)], for $F / d=0.90$, noise level $R=0.05$, and different initial conditions: $x_{0}=0$ (solid line), $x_{0}=0.1$ (dash-dotted line), and $x_{0}=0.2$ (dashed line). We compare the result of the numerical simulations (wiggled lines) with the analytical distribution proposed in the text [Eq. (42)] (smooth lines). This distribution is a weighted sum of the distribution deduced in the high-friction limit [Eq. (29)] and the distribution of early escapes [Eq. (40)], but the relative weight must be adjusted numerically: $c=18.33$ (for $\left.x_{0}=0\right), c=20.62\left(x_{0}=0.1\right)$, and $c=23.34\left(x_{0}=0.2\right)$.

tem with finite dissipation as long as we can calculate: (a) the reinjection time interval $t_{r}$ and (b) the reinjection point $x_{0}$ corresponding to the value of the dissipation $\gamma$. These two values can be obtained numerically integrating the unstable manifold of the saddle, using the determinisic equations. If we now define a narrow two dimensional box as displayed in Fig. 7(c) we can compute the deterministic reinjection time $t_{r}$ and the coordinate of the reinjection point $x_{0}$. Since the unstable manifold of the saddle is attractive, these estimates also apply to the trajectories obtained when small noise is added. Therefore, in order to reproduce the interspike histogram it suffices to evaluate $W_{t o t}\left(x_{0}, t^{\prime}-t_{r}\right)$, where $t^{\prime}$ $=t d / \gamma$ is the rescaled time. Note that our distribution function depends on a somewhat arbitrary choice of the escapebox edge (parameter $b$ ), however this effect is compensated by the variation of the reinjection time $t_{r}$. Typically, $b$ is placed one thermal length $\left(l_{h}=\sqrt{2 R / U^{\prime \prime}\left(x_{n}\right)}\right)$ beyond the saddle.
In Fig. 9 we compare the interspike time distribution for the two dimensional system, close to the homoclinic bifurcation, with the distribution of Eq. (42). This agreement holds for a wide region close to the homoclinic bifurcation (when trajectories are reinjected close the saddle). For intermediate $\gamma$ values, however, the trajectories of the two dimensional system are reinjected between the saddle and the attractor and the one dimensional approach is less effective. For higher values of $\gamma$ the trajectories are reinjected close to the attractor and we return to the high-friction distribution [Eq. (29)] which again gives good agreement with the numerical data.

\section{CONCLUSIONS}

In $[5,4]$ it was reported that noise driven excitable systems could present two characteristic times. It was conjectured that the finite dissipation in the system studied played an

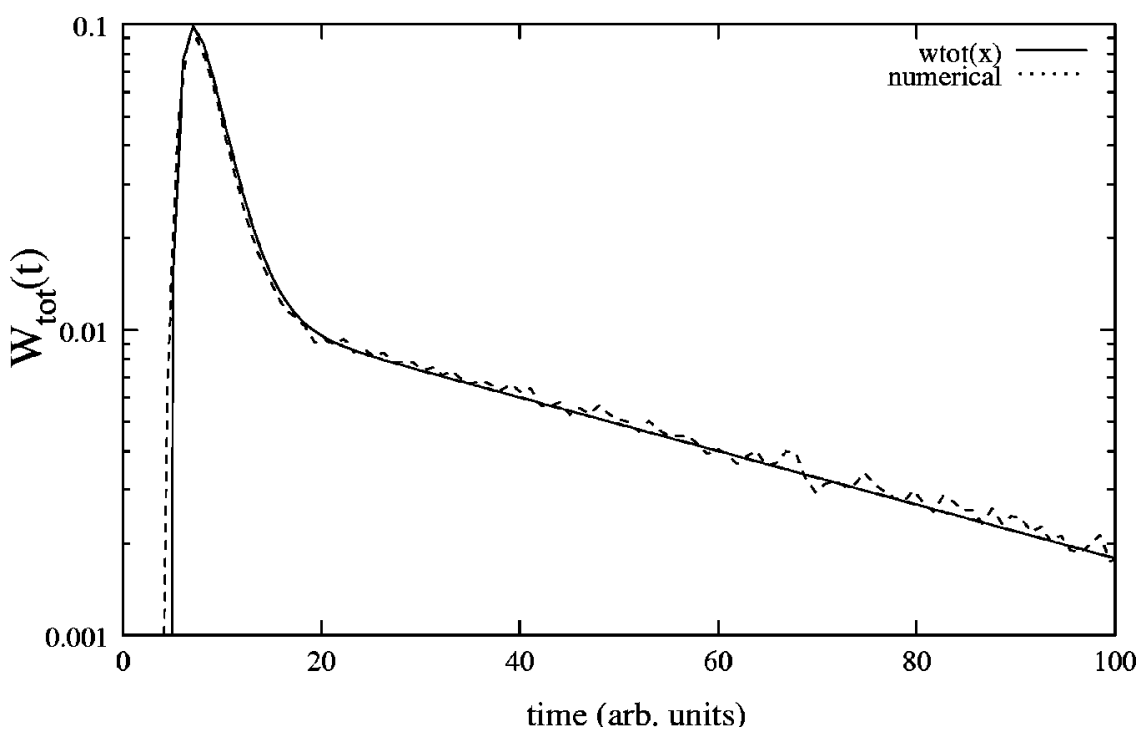

FIG. 9. Distribution of escape times from the attractor for the bidimensional system [Eqs. $(4,6)$ near the homoclinic bifurcation $(F=0.9, d=1.0, \quad \gamma=0.9$, and $D$ $=0.05$ ) obtained from a numerical integration (dashed line) and compared with the approximated analytical distribution function $W_{t o t}\left(x_{0}, t d / \gamma-t_{r}\right)$ [see Eq. (42)]. All parameters but $x_{0}, t_{r}$, and $c$ have analytical expressions. $x_{0}$ and $t_{r}$ can be calculated numerically from the deterministic part of the system and $c$ is the only parameter fitted with the real distribution. For these parameter values $x_{0}=0.3, t_{r}=4.47$, and $c=30.04$. 
important role. In this work we analyzed this problem in detail, studying the evolution of interspike time distributions as the dissipation was changed in a noisy pendulum with torque. We have solved, under a reasonable linear approximation, the high-friction limit problem which has a singlepeaked distribution of escape times. Next, we worked out the finite dissipation problem as a superposition of a two dimensional reinjection process (which has to be solved numerically) and a one dimensional escape process. We found that in this case, the interspike time distribution can be expressed as the sum of two one peaked distributions: the one of the high-friction limit and a distribution of early escapes, both calculated from a linear stochastic process.

These results can be generalized to other systems which present the same dynamical ingredients: a saddle-node bifurcation on a limit cycle and an homoclinic bifurcation, which are organized around a saddle-node-homoclinic bifurcation point. The minimal dimension required to achieve this is two, therefore histograms like the one of Fig. 9 cannot be found in one dimensional excitable systems (Adler equation) and hardly in strong dissipative excitable systems like Fitz-
Hugh Nagumo and Hodgkin-Huxley. On the other hand, there are many two (or more) dimensional unfoldings of the saddle-node-homoclinic bifurcation (like the equations presented in [5]), which have a similar manifold organization (eventually, the two distributions derived in this work might have their maxima separated enough to give rise to a bimodal interspike distributions as in [5]).

Among the systems with the features analyzed in this work, there are also models of excitable neurons (like the Wilson-Cowan [12]), which display interspike histograms qualitatively similar to the ones presented here. This could be especially useful because it is well known that in many neurons, the relevant information is believed to be coded in the inter-spike time distribution [13].

\section{ACKNOWLEDGMENTS}

We thank P. Holmes and H. Vucetich for useful comments. This work was partially funded by Fundación Antorchas, UBA, and CONICET.
[1] P. C. Coullet, S. C. Müller, and D. Walgraef, Chaos 4, 3 (1994).

[2] H. Kramers, Physica (Utrecht) 7, 284 (1940).

[3] P. Hänggi, P. Talkner, and M. Borkovec, Rev. Mod. Phys. 62, 251 (1990).

[4] M. C. Eguia and G. B. Mindlin, Phys. Rev. E 60, 1551 (1999).

[5] A. Yacomotti, M. C. Eguia, J. Aliaga, G. B. Mindlin, O. E. Martinez, and A. Lipsich, Phys. Rev. Lett. 83, 292 (1999).

[6] D. Sigeti and W. Horsthemke, J. Stat. Phys. 54, 1217 (1989).

[7] A. S. Pikovsky and J. Kurths, Phys. Rev. Lett. 78, 775 (1997).

[8] E. Stone and P. Holmes, SIAM (Soc. Ind. Appl. Math.) J. Appl. Math. 50, 726 (1990).

[9] H. Risken, The Fokker-Planck Equation (Springer, Berlin,
1989).

[10] L. Pontryagin, A. Andronov, and A. Vitt, Zh. Eksp. Teor. Fiz. 3, 165 (1933); translated in Noise in Nonlinear Dynamical Systems, edited by F. Moss and M. McClintock (Cambridge University Press, Cambridge, 1989).

[11] W. Feller, An Introduction to Probability Theory and its Applications (Wiley, New York, 1957).

[12] F. C. Hoppensteadt and E. M. Izhikevich, Weakly Connected Neural Networks (Springer, Berlin, 1997).

[13] F. Rieke, D. Warland, R. de Ruyter van Steveninck, and W. Bialek, Spikes: Exploring the Neural Code (MIT Press, Cambridge, MA, 1997). 